\title{
The Evaluation of Emergency Response Capability for Metro Construction Based on CUOWA Operator
}

\author{
Jinxian Zhao ${ }^{1, a}$,Jinghan Sun ${ }^{1, b}$, Ke Fan $^{1, \mathrm{c}}$,Min Liu ${ }^{1, \mathrm{~d}}$ \\ ${ }^{1}$ School of Management,Qingdao Technological University, Qingdao 266520,China

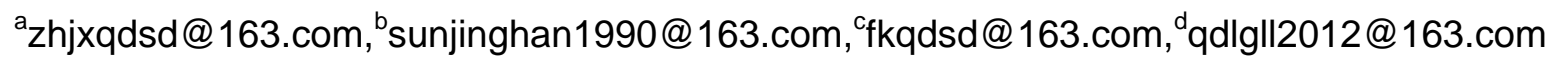

\begin{abstract}
Keywords: Metro construction;emergency response capability;index system;OWA operator;evaluation methodology.

Abstract.According to the characteristics during construction of the metro incidents, an emergency capability evaluation index system of metro construction was established in the paper,which based on the relevant theory of the ability of emergency management. Build up a metro construction of emergency capability evaluation modelbased on the Combined Uncertain OWA(referred to as the CUOWA) operator ,by using improved analytic hierarchy process (AHP) to determine the index weight.Finally, the evaluation model is applied to the concrete examples, and some suggestions are put forward to strengthen the emergency response capability, which can provide reference for the evaluation of emergency response capability in the metro construction process.
\end{abstract}

\section{Introduction}

While emergencies are occurring in the process of metro construction, a series of major accidents caused by the collapse of the metro,such as Beijing, Shanghai, not only caused significant casualties, but also caused a certain social panic.This has to make us reflect on theability and levelof emergency management in metro construction projects in our country.

At present, researches on metro emergency management in china are numerous.Such asNational Disposal of Urban MetroAccident Disaster Emergency Planpublished by the state council [1]; one emergency management research is conducted combining the characteristics of metro emergencies and the actual situation of metro [2]; one designed an information system of metro emergency management,established an overall architecture of the system platform [3];one analyzed the city metro emergency plan system and proposed measures to improve the urban metro emergency plan [4]. With the research of emergency response capability on metro emergency management focusing on the operational phase, this article mainly aims at the characteristics of the metro emergencies in the process of construction in our country, using the method of CUOWA operator to evaluate the level of emergency response capability.

\section{The Index System construction of metro construction for emergency response capability}

According to the characteristics of the metro emergencies in the process of construction in our country, the metro construction emergency capability evaluation systemwas builtcombiningthe causes of domestic and home underground engineeringaccidents and aspects ofmetro construction process and potential hazards,coveringfour aspects like emergency preparation, early warning capability, rescue capability, recovery and reconstruction, including 4 secondary indicators and 20 three-grade indicators, which are shown inFig. 1. 


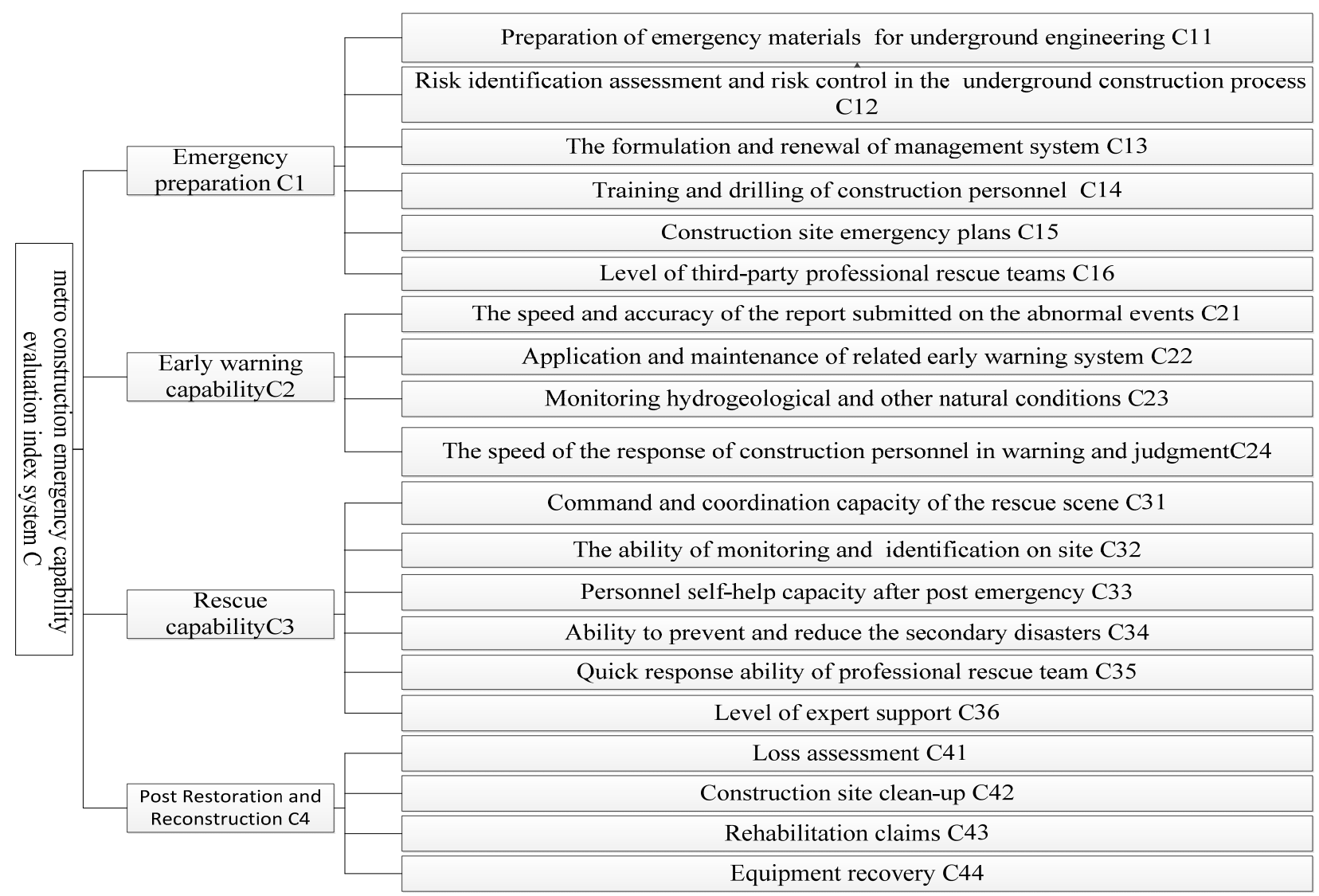

Fig. 1The emergency capability evaluation index system

\section{Evaluation model of emergency response capability for Metro Construction Based on CUOWA operator}

\section{Determination of index weight}

The twoor three level index weights are determined by using the theory of AHP (analytic hierarchy process), which takes full advantage of the experience analysis, judgment and comprehensive ability, and makes quantitative analysis of the qualitative index factor[5].

In order to avoid the huge difference between the weight of the same level indicators, prevent to impact the superiority of CUOWA operator, this paper uses the following method to improve the weight, that is, the weight is greater than 0.3 of the index to cut, will share the parts of it to the other indicators, and finally get the weight of each index.

$$
w_{i}^{*}=w_{i}+w_{i} / \sum w_{k} \times\left(w_{j}-0.3\right)
$$

In the type,$w_{i} *$ is Modified index weight, $\mathrm{w}_{\mathrm{i}}$ isindex weight before modified, $w_{j}$ is the Weight greater than the index weight of $0.3, w_{k}=1-w_{j}$.

\section{Constructing the evaluation model of CUOWA operator \\ Determine the comprehensive measures of emergency response capability for Metro Construction}

In the decision-making process, the CUOWA operator is used to evaluate the index system, avoid the uncertainty of decision environment andincomplete information getting from the decision group (people)and other characteristics, so the decision-making problem is transformed from qualitative to quantitative[6]

Assumed attributeA $=\{$ Emergency response capability of Metro Construction $\}$, Divide it into four levels : $B_{1}=\{$ Excellent $\}, B_{2}=\{\operatorname{good}\}, B_{3}=\{$ qualified $\}, B_{4}=\{$ poor $\},\left(B_{1}, B_{2}, B_{3}, B_{4}\right)$, To form an ordered partition class $A$, The ordered partition class is in the range of $[0,10]$, The specific classification are shown in Table 1. 
Table 1 Comprehensive measure of the level of emergency response capability for Metro Construction

\begin{tabular}{c|c|c|c|c}
\hline grades & $\mathrm{B}_{1}$ & $\mathrm{~B}_{2}$ & $\mathrm{~B}_{3}$ & $\mathrm{~B}_{4}$ \\
\hline measure interval & $(9,10]$ & $(7,9]$ & $(5,7]$ & {$[0,5]$} \\
\hline
\end{tabular}

Quantitative fuzzy semantics

In the study of decision-making system, the fuzzy semantic mainly including "Majority", "A t least half", "As much as possible", Theirrespective parameters are respectively $(a, b)=(0.3,0.8),(a, b)=(0,0.5),(a, b)=(0.5,1)$.

According to the above fuzzy semantic criteria, select the appropriate fuzzy semantics, computing parameters $(a, b)$ CUOWA Weighted vector $w=\left(w_{1}, w_{2}, \cdots, w_{n}\right)$ :

$w_{j}=Q(j / n)-Q((j-1) / n), \quad j \in N(2)$

Among them, $Q$ is a fuzzy semantic quantization operator, the formula is as follows:

$Q(r)=\left\{\begin{array}{ccc}0, & r<a \\ (r-a) /(b-a) & , a \leq r \leq b \\ 1, & r>b & \end{array}\right.$

\section{Evaluation process based on CUOWA operator}

Combined with comprehensive measures of emergency response capability for metro construction, invited $m$ experts to evaluate the emergency ability of emergency capability evaluation index system of evaluation, taking the lowest scores and the highest score decision to form the range of indicators,the $n$ indicators $C_{i j}$ of a group decision interval constitute decision $\operatorname{data}\left(\tilde{a}_{1}, \tilde{a}_{2}, \cdots, \tilde{a}_{n}\right)$.

(1)A new decision data set is obtained by combining the weights of the index weights $\omega_{i}$ and the number of $n$ and the $\left(\tilde{a}_{1}, \tilde{a}_{2}, \cdots, \tilde{a}_{n}\right)$ of the evaluation group. $\tilde{a}^{\prime}=\left(n \omega_{1} \tilde{a}_{1}, n \omega_{2} \tilde{a}_{2}, \cdots, n \omega_{n} \tilde{a}_{n}\right)$

(2)The decision-making data $\tilde{a}_{j}^{\prime}$ a pairwise comparison, may build degree matrix $P=\left(p_{i j}\right)_{n \times n}$, $p_{i j}=p\left(\tilde{a}_{i}^{\prime} \geq \tilde{a}_{j}^{\prime}\right)=p\left(n \omega_{i} \tilde{a}_{i} \geq n \omega_{j} \tilde{a}_{j}\right)$, the degrees are defined as follows: Assuming that $\tilde{b}=\left[b^{L}, b^{U}\right]$, make $l_{\tilde{a}}=a^{U}-a^{L}, l_{\tilde{b}}=b^{U}-b^{L}$, so the possibility of interval number $\tilde{a} \geq \tilde{b}$ is $p(\tilde{a} \geq \tilde{b})=\frac{\min \left[l_{\tilde{a}}+l_{\tilde{b}}, \max \left(0, a^{U}-b^{L}\right)\right]}{l_{\tilde{a}}+l_{\tilde{b}}}$

(3)According to the previous step to get the possible degrees of matrix calculation of compl ementary judgment matrix

$v_{i}=\frac{1}{n(n-1)}\left(\sum_{j=1}^{n} p_{i j}+\frac{n}{2}-1\right), \quad i \in N$

Get sorted vector $v=\left(v_{1}, v_{2}, \cdots, v_{n}\right)^{T}$

(4)The decision data $\tilde{a}^{\prime}=\left(n \omega_{1} \tilde{a}_{1}, n \omega_{2} \tilde{a}_{2}, \cdots, n \omega_{n} \tilde{a}_{n}\right)$ is sorted according to the order of $v_{i}$. The data set is obtained from the large to small data $\tilde{b}_{j}$. Using the fuzzy semantic quantization matrix $w$ to carry on the weighted operation to the data set $\tilde{b}$, CUOWA operator is

CUOWA $_{\omega w}\left(\tilde{a}_{1}, \tilde{a}_{2}, \cdots, \tilde{a}_{n}\right)=\sum_{j=1}^{n} w_{j} \tilde{b}_{j}$

In this paper, the underlying index weighted synthetic CUOWA operator according to the above steps as the comprehensive evaluation of the index of the upper level, and the upper index weighted assembled again get more on a layer of comprehensive evaluation of the level.Since CUOWA 
operator is an interval number, in order to make the evaluation result straightforward, you can take the number between intermediate values as the final evaluation results.To determine the key improve object by the evaluation results, make effective improvement strategies.

\section{Case analysis}

\section{Index weight calculation}

Taking a metro construction section engineering as an example, the construction emergency response capability is evaluated, invited the industry experts to compare the importance of each index between two means to get the judgment matrixcalculate the weight of each index by using AHP. After using Eq.1 to modify its again,finallyobtaining the characteristic vector for $W_{1}=\left[\begin{array}{llllll}0.109 & 0.25 & 0.214 & 0.13 & 0.179 & 0.118\end{array}\right]^{T}, W_{2}=\left[\begin{array}{lllll}0.257 & 0.3 & 0.3 & 0.143\end{array}\right]^{T}$,

$$
W_{3}=\left[\begin{array}{llllll}
0.174 & 0.109 & 0.219 & 0.152 & 0.201 & 0.145
\end{array}\right]^{T}, W_{4}=\left[\begin{array}{llll}
0.257 & 0.3 & 0.143 & 0.3
\end{array}\right]^{T} \text {. }
$$

\section{CUOWA operator evaluation}

Invited eight experts participated in the construction of metro construction section evaluate the evaluation system,take the index of emergency preparation as an example, it decision-making data are shown in Table 2.

Table2 expert evaluation value for $C_{11} \sim C_{16}$

\begin{tabular}{c|c|c|c|c|c|c|c|c}
\hline Index & Expert1 & Expert2 & Expert3 & Expert4 & Expert5 & Expert6 & Expert7 & Expert8 \\
\hline$C_{11}$ & 7.5 & 8.5 & 9.5 & 7.5 & 7.5 & 8.5 & 7 & 7.5 \\
\hline$C_{12}$ & 7 & 8 & 7.5 & 6 & 5.5 & 6.5 & 8.5 & 7 \\
\hline$C_{13}$ & 8 & 8.5 & 10 & 9 & 8.5 & 9.5 & 8 & 9 \\
\hline$C_{14}$ & 8 & 8.5 & 9.5 & 8.5 & 8.5 & 9.5 & 8 & 8 \\
\hline$C_{15}$ & 9 & 7 & 9 & 10 & 8 & 7 & 9.5 & 7.5 \\
\hline$C_{16}$ & 10 & 8.5 & 8 & 8 & 9 & 8.5 & 9 & 8.5 \\
\hline
\end{tabular}

Thedecision data interval number is, $a_{11}=\left[\begin{array}{ll}7.5 & 9.5\end{array}\right], a_{12}=\left[\begin{array}{ll}5.5 & 8.5\end{array}\right], a_{13}=\left[\begin{array}{ll}8 & 10\end{array}\right], a_{14}=\left[\begin{array}{ll}8 & 9.5\end{array}\right]$, $a_{15}=\left[\begin{array}{ll}7 & 10\end{array}\right], a_{16}=\left[\begin{array}{ll}8 & 10\end{array}\right]$. The index weight, the index number and the decision data are combined toobtain the new decision data group $\quad n a_{1} \omega_{1}=[4.905,6.213], n a_{2} \omega_{2}=[8.25,12.75]$, $n a_{3} \omega_{3}=[10.272,12.84], n a_{4} \omega_{4}=[6.24,7.41], n a_{5} \omega_{5}=[7.518,10.74], n a_{6} \omega_{6}=[5.664,7.08]$. According to the Eq.4 possible degree matrix are obtained:

$P=\left[\begin{array}{cccccc}0.5 & 0 & 0 & 0 & 0 & 0.202 \\ 1 & 0.5 & 0.351 & 1 & 0.678 & 1 \\ 1 & 00.649 & 0.5 & 1 & 0.904 & 1 \\ 1 & 0 & 0 & 0.5 & 0 & 0.675 \\ 1 & 0.322 & 0.096 & 1 & 0.5 & 1 \\ 0.798 & 0 & 0 & 0.325 & 0 & 0.5\end{array}\right]$

According to the Eq. 5 sorting vector calculation available indicators $v=(0.09,0.218,0.235,0.139,0.197,0.121)$

Get sorted vector $b_{1}=[10.272,12.84], b_{2}=[8.25,12.75], b_{3}=[7.518,10.74], b_{4}=[6.24,7.41], b_{5}=$ $[5.664,7.08], b_{6}=[4.905,6.213]$.In this paper, the fuzzy evaluation by default "as much as possible",According to the Eq.2,3 can get the weighted vector $w=(0,0,0,0.333,0.333,0.333)$.Accor ding to the Eq.6, the comprehensive evaluation value of the index $\quad C_{1}, C_{2}, C_{3}, C_{4}$ can

be obtained CUOWA $=[5.603,6.901], C U O W A_{2}=[4.972,7.486], C U O W A_{3}=[6.047,7.816], C U O W A_{4}$ 
$=[5.229,7.486]$. For the median calculation, get $U_{1}=(5.603+6.901) / 2=6.252, U_{2}=6.229$, $U_{3}=6.932, U_{4}=6.358$.

Finally, the comprehensive evaluation of the emergency response capability for metro construction is obtained $U=6.64$, Level of emergency ability for metro construction is qualified.

\section{Conclusion}

(1)Through case analysis, the final result is qualified, that is, the level of emergency response capability in the metro construction section of the construction process to meet the basic requirements. Further analysis found that the level of the early warning ability is low in two aspects. including the speed and accuracy of the report submitted on the abnormal events and the speed of the response of construction personnel in warning and judgment, as well as that in emergency preparation, the evaluation score is low in onsite emergency supply preparation and third-party professional rescue teams. Therefore, these specific indicators in the process of metro construction should be enhanced and improved one by one in the future.

(2)This paper has established the emergency capability evaluation model of metro construction, using the method of improved AHP and CUOWA operator quantitative to qualitative problem, the characteristics of the evaluation model is simple and practical. It have a higher level of value for evaluating emergency response capability of metro construction process. If it is improved, it can be applied to other large engineering evaluation process of emergency response capability,

\section{References}

[1] HUANG.W.J, LI.C.G, Assessment of Emergency capacity to Major Incidents on Urban Subway,(2006).

[2] LU.W.G,PENG.J, The Study of Capability Assessment for metro Emergency System on Guangzhou, Urban Studies.(2012),04:118-124.

[3] WANG.Q.K,LIU.K.Y, Design of Emergency Management Information System for Metro Engineering Construction, C Journal of Huazhong University of Science and Technology, (2011),02:67-72.

[4] Han. J: Research on the Emergency Preparedness Plans of the Urban metro (2010)

[5] Du.D, Pang.Q.H, Wu.Y,Modern evaluation methods and case selection,Beijing,(2012)

[6]ZHOU.L.G,CHEN.H.Y,WU.X.F,WEI.D, A Method of Interval Number Group Decision Making Based on the Combined Uncertain OWA Operator, College Mathematics ,(2011),02:52-56. 